\title{
The influence of phonons on the optical properties of GaN
}

\author{
D. Y. Song, M. Basavaraj, S. A. Nikishin, and M. Holtz ${ }^{\mathrm{a})}$ \\ Texas Tech University, Lubbock, Texas 79409 \\ V. Soukhoveev, A. Usikov, and V. Dmitriev \\ TDI, Inc., 12214 Plum Orchard Drive, Silver Spring, Maryland 20904
}

(Received 4 May 2006; accepted 10 August 2006; published online 1 December 2006)

\begin{abstract}
We comprehensively examine the importance of phonons on the optical properties of GaN. Using Raman and photoluminescence (PL) spectroscopies, the energies and linewidths of optic phonons, excitons, and discrete phonon sidebands (PSBs) are studied between 20 and $325 \mathrm{~K}$. The temperature dependence of the $\mathrm{A}_{1}(\mathrm{LO})$ phonon energy and linewidth are described by a combined two- and three-phonon decay process. The narrow $E_{2}^{2}$ phonon decays by the three-phonon emission process. Three band-edge excitons are observed in PL with linewidths between 2.8 and $5.3 \mathrm{meV}$ at temperature $22 \mathrm{~K}$. The energy gap shrinkage and exciton linewidths are completely interpreted based on electron-phonon interactions. The shift, broadening, and asymmetry of the PSBs are explained by incorporating the decay mechanism of $\mathrm{A}_{1}(\mathrm{LO})$ phonon and the exciton broadening from electron-phonon interactions. (c) 2006 American Institute of Physics.
\end{abstract}

[DOI: $10.1063 / 1.2361159]$

\section{INTRODUCTION}

The lifetimes of intrinsic phonons in high-quality crystalline materials are determined by phonon decay processes. ${ }^{1,2}$ The decay mechanisms are governed by energy and wave vector conservation, and specific decay channels are important when those vibrations created have high density of states. Phonon decay is important to understand in materials when devices based on them exhibit self heating, such as GaN-based bipolar and field-effect transistors. ${ }^{3} \mathrm{Op}$ tical properties of semiconductors are useful for understanding the electronic band structure. This is particularly important for states near the valence and conduction edges which are the most relevant to light emitting devices and to carrier transport. The band structure and behavior of carriers in such materials are strongly influenced by lattice vibrations. For direct optical transitions the zone-center phonons are most relevant. In the III-nitride semiconductors, e.g., GaN, bulk crystals are not widely available. Consequently, studies of these semiconductors have primarily relied on heteroepitaxy using foreign substrates. ${ }^{4,5}$ The resultant epitaxial crystals are generally abundant in dislocations. This situation is in stark contrast to other semiconductors for which high-quality bulk crystals are routinely available, and hinders study of intrinsic $\mathrm{GaN}$ properties.

Recent advances in growing epitaxial GaN, with relatively low defect density, ${ }^{6}$ present the opportunity to study fundamental optical processes across a broad temperature range. Narrow phonon lines observed in high-quality crystals are likewise necessary to examine the decay properties of the zone-center phonons of GaN. The decay is examined through temperature dependence of the Raman-active $\mathbf{k}=0$ phonon energy and linewidth. GaN crystals with low dislocation den-

\footnotetext{
a) Author to whom correspondence should be addressed; electronic mail: Mark.Holtz@ttu.edu
}

sity enable examination of the band-edge excitons, through photoluminescence (PL) spectroscopy, and the properties of discrete $\mathrm{A}_{1}$ (LO) phonon sidebands (PSBs).

We present a study of phonon decay and the influence of phonons on excitons and the band gap of GaN. Using Raman scattering, we study the decay properties of $E_{2}^{2}$ and longitudinal-optic $\mathrm{A}_{1}$ (LO) symmetry phonons. We also examine the near-band gap PL to study the temperature dependence of the $\mathrm{GaN}$ energy gap, via the exciton emission, and their $A_{1}$ (LO) PSBs. The band gap shrinkage, the exciton linewidth, and the PSBs are all strongly dependent on the vibrational properties. The results are remarkably consistent, providing a comprehensive examination of the impact phonons have on the optical properties of GaN.

\section{EXPERIMENTAL DETAILS}

The nominally undoped GaN layers, with layer thickness $\sim 80 \mu \mathrm{m}$, were grown using hydride vapor phase epitaxy on 2-in. sapphire substrates. ${ }^{7}$ The layers had smooth surface morphology with root-mean-square (rms) roughness below $0.5 \mathrm{~nm}$ as measured by atomic force microscopy using 5 $\times 5 \mu \mathrm{m}^{2}$ scans. No cracks were observed. The concentration of electrically active (noncompensated) donors was in the low $10^{16} \mathrm{~cm}^{-3}$ range from capacitance-voltage measurements. X-ray diffraction reveals layers to have low dislocation density, $<10^{8} \mathrm{~cm}^{-2}$, and to be strain relaxed. ${ }^{8}$

Micro-Raman spectra were measured in a backscattering geometry using excitation wavelength $488.0 \mathrm{~nm} .{ }^{9}$ Micro-PL spectra were obtained with $351.0 \mathrm{~nm}$ excitation and dispersed using a $0.78 \mathrm{~m}$ double monochromator. Linewidths have been corrected for the instrumental bandpass. Excitation power was kept well below $10^{4} \mathrm{~W} / \mathrm{cm}^{2}$ to minimize local heating. Temperature was controlled between $\sim 20$ and $325 \mathrm{~K}$ using a closed-cycle cryostat. In this article we use the conventional notation of $\omega$ for phonon energy in $\mathrm{cm}^{-1}$ and $\varepsilon$ for energy in electron-volts or milli-electron-volts. 


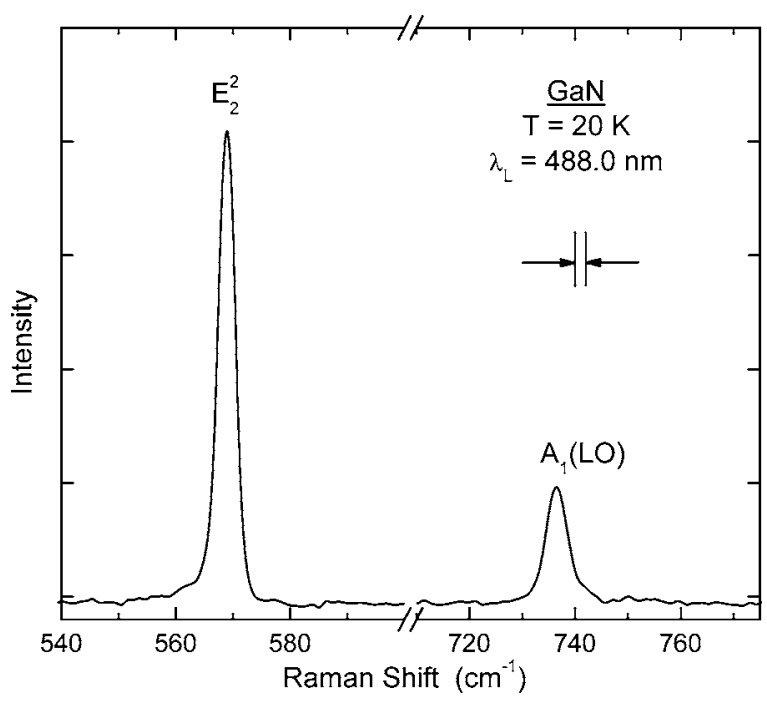

FIG. 1. Raman spectrum of GaN epilayer at $20 \mathrm{~K}$.

\section{III. $E_{2}^{2}$ AND $A_{1}$ (LO) PHONONS}

The Raman spectrum for the GaN sample at $T=20 \mathrm{~K}$ is shown in Fig. 1. The Raman shift and linewidth ( $\Gamma$, full width at half maximum) are determined by fitting Lorentzian functions to the data. Two features at 569 and $737 \mathrm{~cm}^{-1}$ correspond to the $E_{2}^{2}$ and $\mathrm{A}_{1}$ (LO) phonons. ${ }^{10}$ The $E_{2}^{2}$ and $\mathrm{A}_{1}(\mathrm{LO})$ intensities are as expected for $\mathrm{GaN}$ with low free-carrier concentration. ${ }^{11}$ The linewidths of $E_{2}^{2}$ and $\mathrm{A}_{1}$ (LO) at $T=20 \mathrm{~K}$ compare well with previously published reports ${ }^{12-15}$ indicating high crystalline quality material.

In Fig. 2 we show the temperature dependences of the phonon energies and linewidths. The Raman bands redshift with temperature and broaden, although the changes are stronger for the $\mathrm{A}_{1}(\mathrm{LO})$ line than for $E_{2}^{2}$. The temperature dependences of $\omega(T)$ can be described by the crystal thermal expansion, anharmonic interactions, and lattice and thermal mismatches between the substrate and the epitaxial layer

$$
\omega(T)=\omega_{0}-\Delta_{1}(T)-\Delta_{2}(T),
$$

where $\omega_{0}$ is the harmonic frequency of the phonon mode, $\Delta_{1}(T)$ is the thermal expansion contribution to the frequency, and $\Delta_{2}(T)$ is the anharmonic interaction term. The thermal strain is negligible $\left[<0.1 \mathrm{~cm}^{-1}\right.$ shift for the $\mathrm{A}_{1}(\mathrm{LO})$ phonon] across the temperature range studied. ${ }^{16}$ The thermal expansion contribution is given by $\Delta_{1}(T)=\omega_{0} \gamma \int_{0}^{T}\left[\alpha_{c}\left(T^{\prime}\right)\right.$ $\left.+2 \alpha_{a}\left(T^{\prime}\right)\right] d T^{\prime}$, where $\gamma$ is the Gruneisen parameter, and $\alpha_{c}$ and $\alpha_{a}$ are the temperature-dependent linear thermal expansion coefficients parallel and perpendicular to the hexagonal $c$ axis, respectively. ${ }^{17}$ The anharmonic shift $\left.\Delta_{2}(T)\right)$ is ${ }^{16}$

$$
\begin{aligned}
\Delta_{2}(T)= & A\left[1+n\left(\omega_{1}, T\right)+n\left(\omega_{2}, T\right)\right] \\
& +B\left[1+n\left(\omega_{3}, T\right)+n\left(\omega_{4}, T\right)+n\left(\omega_{5}, T\right)\right. \\
& \left.+n^{2}\left(\omega_{3}, T\right)+n^{2}\left(\omega_{4}, T\right)+n^{2}\left(\omega_{5}, T\right)\right],
\end{aligned}
$$

where $n(\omega, T)=\left[\exp \left(h c \omega / k_{B} T\right)-1\right]^{-1}$ is the Bose function at energy $h c \omega$. The first term in Eq. (2) corresponds to the two-phonon decay process, where $\omega_{1}$ and $\omega_{2}$ are frequencies of the phonons created by the decay. The second term in Eq. (2) corresponds to the three-phonon decay process with en-
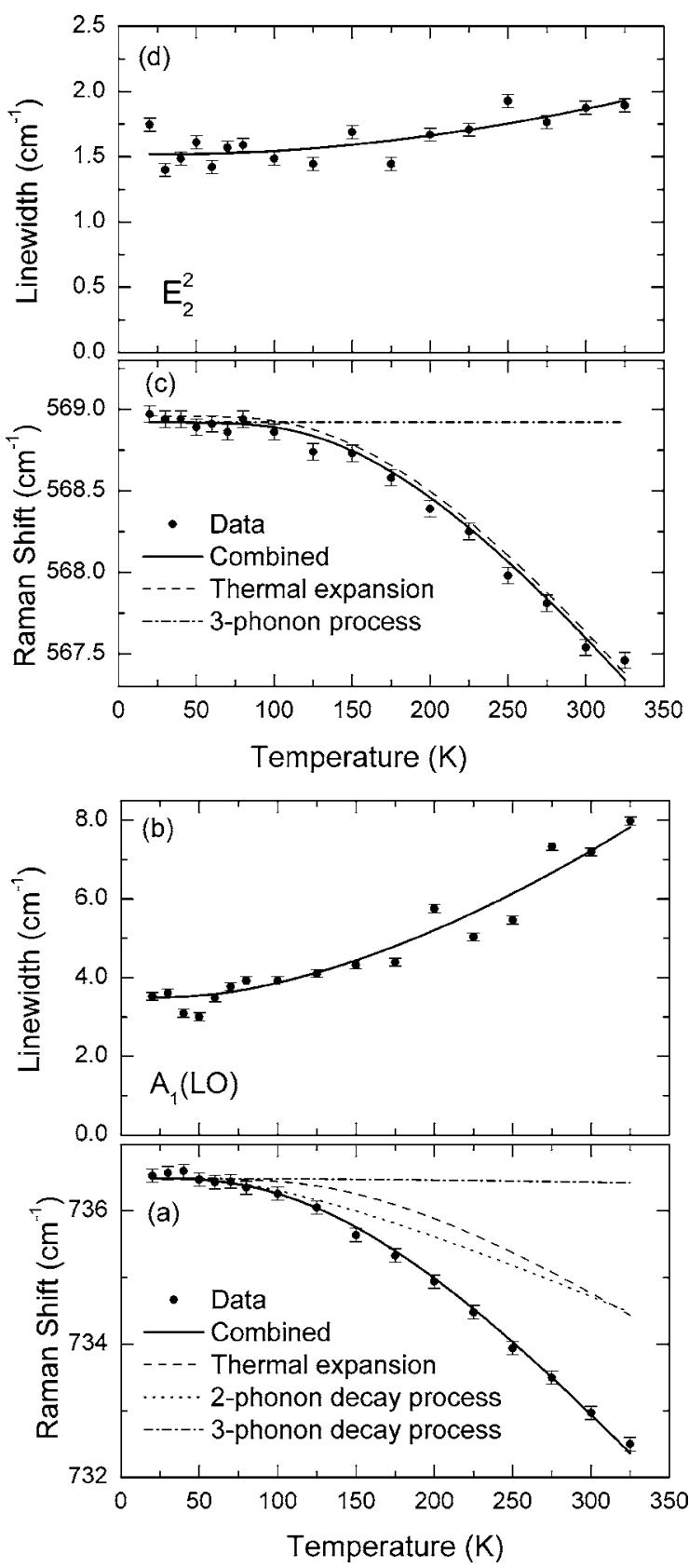

FIG. 2. Dependence of Raman-active phonon energies and linewidths on temperature: (a) and (b) are for $\mathrm{A}_{1}(\mathrm{LO}),(\mathrm{c})$ and (d) are for $E_{2}^{2}$. Curves are fits to data. Different contributions to the phonon shifts are plotted separately in (a) and (c), respectively.

ergies $\omega_{3}, \omega_{4}$, and $\omega_{5}$. The created/annihilated phonon energies must satisfy conservation of energy. For the zone-center Raman-active phonons, crystal momentum conservation requires the sum of the wave vectors of the created phonons in the decay to be zero. Anharmonic constants $A$ and $B$ identify the relative probabilities of the decay processes.

The Raman linewidth can be described in terms of anharmonic interactions ${ }^{18}$

$$
\Gamma_{P}(T)=\Gamma_{0}+\Delta_{2}^{\prime}(T),
$$

where $\Delta_{2}^{\prime}(T)$ is identical in form to $\Delta_{2}(T)$ with $A(B)$ replaced by $C(D)$, and the $\omega_{i}$ energies are the same as what we obtain from fitting Eq. (2) to the phonon energy data. With 
TABLE I. Fitting results for Raman phonons and band gap related excitons All $T=0 \mathrm{~K}$ values are from fits to the temperature dependences.

\begin{tabular}{|c|c|c|}
\hline Excitation & Energy & Linewidth \\
\hline$\overline{E_{2}^{2}}$ & $\begin{array}{l}\omega_{0}=569.1 \mathrm{~cm}^{-1} \\
A=0, B=0.09 \mathrm{~cm}^{-1} \\
\omega_{3}=\omega_{4}=\omega_{5}=190 \mathrm{~cm}^{-1}\end{array}$ & $\begin{array}{l}\Gamma_{0}=1.4 \mathrm{~cm}^{-1} \\
C=0, D=0.10\end{array}$ \\
\hline $\mathrm{A}_{1}(\mathrm{LO})$ & $\begin{array}{l}\omega_{0}=738.7 \mathrm{~cm}^{-1} \\
A=2.2 \mathrm{~cm}^{-1}, B=0.09 \mathrm{~cm}^{-1} \\
\omega_{1}=\omega_{3}=559 \mathrm{~cm}^{-1}, \omega_{2}=180 \mathrm{~cm}^{-1} \\
\omega_{4}=\omega_{5}=90 \mathrm{~cm}^{-1}\end{array}$ & $\begin{array}{l}\Gamma_{0}=1.4 \mathrm{~cm}^{-1} \\
C=1.9 \mathrm{~cm}^{-1}, D=0.2 \mathrm{~cm}^{-1}\end{array}$ \\
\hline$X_{A}$ & $\begin{array}{l}E(0)=3.473 \mathrm{eV} \\
\varepsilon_{a c}=22.5 \mathrm{meV}=181 \mathrm{~cm}^{-1} \\
\varepsilon_{\mathrm{opt}}=69.3 \mathrm{meV}=559 \mathrm{~cm}^{-1} \\
W_{a c}=0.49, W_{\mathrm{opt}}=0.51\end{array}$ & $\begin{array}{l}\Gamma(0)=5.8 \mathrm{meV}=47 \mathrm{~cm}^{-1} \\
\Gamma_{a c}=50 \mathrm{meV}=400 \mathrm{~cm}^{-1} \\
\Gamma_{\mathrm{opt}}=110 \mathrm{meV}=890 \mathrm{~cm}^{-1}\end{array}$ \\
\hline$X_{B}$ & $E(0)=3.480 \mathrm{eV}$ & \\
\hline$\left(D^{0}, X\right)$ & $E(0)=3.467 \mathrm{eV}$ & $\Gamma(0)=3.7 \mathrm{meV}=30 \mathrm{~cm}^{-1}$ \\
\hline
\end{tabular}

these constraints, the number of fitting parameters inherent in the phonon-decay model is held to a minimum. $\Gamma_{0}$ is related to the phonon lifetime and is affected by inherent disorder or impurity scattering in high-quality material. If we attribute the $\mathrm{A}_{1}$ (LO) phonon linewidth to homogeneous broadening only, the lifetime obtained is $\sim 1.6 \mathrm{ps}$ which compares well with measurements using time-resolved spectroscopy. ${ }^{19}$

We use Eqs. (1) and (3) to fit the dependence of our $\omega(T)$ and $\omega(T)$ data, as shown in Figs. 2(a) and 2(b). Fit results are included in each panel and parameters are summarized in Table I. For the Raman energy we show the individual contributions of the thermal expansion, Eq. (2) shifted by $\omega_{0}$, and two-phonon decay process. These terms produce comparable contributions to the overall shift. The peak energy and linewidth results consistently show that two-phonon decay plays a larger role than the three-phonon mechanism in the $\mathrm{A}_{1}$ (LO) phonon temperature dependence $(A \gg B$ and $C \gg D)$. The two-phonon energies 559 and $180 \mathrm{~cm}^{-1}$ are consistent with $\mathrm{LO} \rightarrow \mathrm{TO}+\mathrm{LA}(\mathrm{TA})$ decay. $^{20,21}$ This decay is referred to as the Ridley process. The three-phonon decay energies 560, 90 , and $90 \mathrm{~cm}^{-1}$ are consistent with the $\mathrm{LO} \rightarrow \mathrm{TO}+\mathrm{LA}(\mathrm{TA})$ $+\mathrm{LA}(\mathrm{TA})$ three-phonon decay in Refs. 2 and 21. The twophonon decay is dominant in the temperature dependence of the $\mathrm{A}_{1}(\mathrm{LO})$ phonon behavior.

In contrast to the $\mathrm{A}_{1}(\mathrm{LO})$ phonon, results in Fig. 2(c) show the $E_{2}^{2}$ energy to depend weakly on temperature, redshifting by only $\sim 1.5 \mathrm{~cm}^{-1}$ across the temperature range studied. This is attributed to the absence of two-phonon decay channels satisfying both conservation of energy and wave vector criteria and exhibiting appreciable density of states. Thus, $A=0$ in Eq. (2) for the $E_{2}^{2}$ phonon. In Fig. 2(c) we plot the individual contributions from the dominant thermal expansion and the three-phonon decay process to the overall Raman shift for $E_{2}^{2}$ mode. ${ }^{14,22}$ The decay creates three acoustic phonons at $\sim 190 \mathrm{~cm}^{-1}$. ${ }^{13}$ The linewidth in Fig. 2(d) also varies weakly with temperature, consistent with threephonon decay. The dependence is fitted well with $C=0$ and $D \neq 0$ and using the same decay energies. Our results show

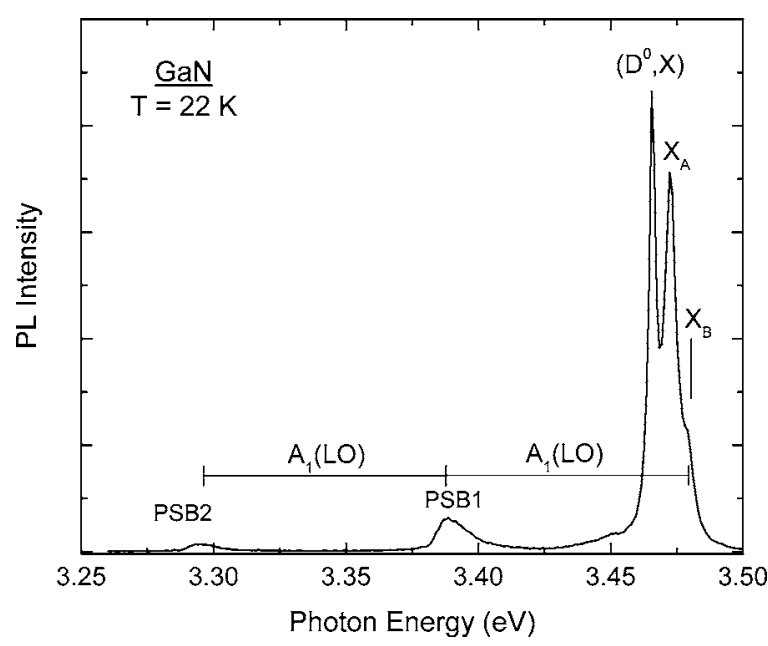

FIG. 3. Photoluminescence spectrum of $\mathrm{GaN}$ epilayer at $22 \mathrm{~K}$. Donor-bound exciton line labeled $\left(D^{0}, X\right), X_{A}$ and $X_{B}$ are free excitons. PSB1 and PSB2 denote $\mathrm{A}_{1}$ (LO)-phonon sidebands.

the contribution from three-phonon decay is very small throughout this temperature range. This is in contrast to previous Raman work, which concluded that the thermal expansion and three-phonon process made comparable contributions. $^{14,22}$ It is important to note that we analyze our phonon energy and linewidth data and that we use the temperature dependent expansion coefficients, which vary significantly over the range of our experiments. ${ }^{17}$

\section{BAND-EDGE EXCITONS}

The phonon properties are critical in understanding the temperature dependence of the energy gap. In Fig. 3 the lowtemperature PL spectrum exhibits three exciton bands at $3.468,3.475$, and $3.482 \mathrm{eV}$. Our exciton lines agree with those of unstrained materials, as expected from our x-ray measurements, identifying these features as the shallow neutral donor-bound $\left(D^{0}, X\right)$ and free excitons $\left(X_{A}\right.$ and $\left.X_{B}\right)$. $^{23}$ Each line is narrow at this temperature, 2.8, 5.3, and $4.7 \mathrm{meV}$, respectively. We see two $\mathrm{A}_{1}$ (LO) PSBs, originating from the $X_{B}$ exciton, with the characteristic asymmetry attributed to interactions between discrete and continuum states. $^{24}$

In Fig. 4(a) we summarize the exciton peak energies as a function of temperature. The characteristic redshift with increasing temperature is attributed to band gap reduction from lattice expansion and to electron-phonon interactions. ${ }^{25}$ Both factors are closely associated with phonon populations. We apply the approach of Pässler, ${ }^{26}$ which incorporates both self-energy Fan $^{27}$ and Debye-Waller ${ }^{28}$ factors. The resulting expression for the temperature shift of the band gap is $\Delta E_{e-p}(T) \rightarrow-\left(\delta / k_{B}\right) \sum_{i=1}^{N} \varepsilon_{i} W_{i} n\left(\varepsilon_{i}, T\right)$, where $\delta$ is the hightemperature linear slope of the band gap, and the weighting factor is normalized $\sum_{i=1}^{N} W_{i}=1$. We adopt a simple twooscillator $(N=2)$ model, corresponding to acoustic and optic phonon branches, and consider the contribution from thermal expansion. The temperature dependence is thus 

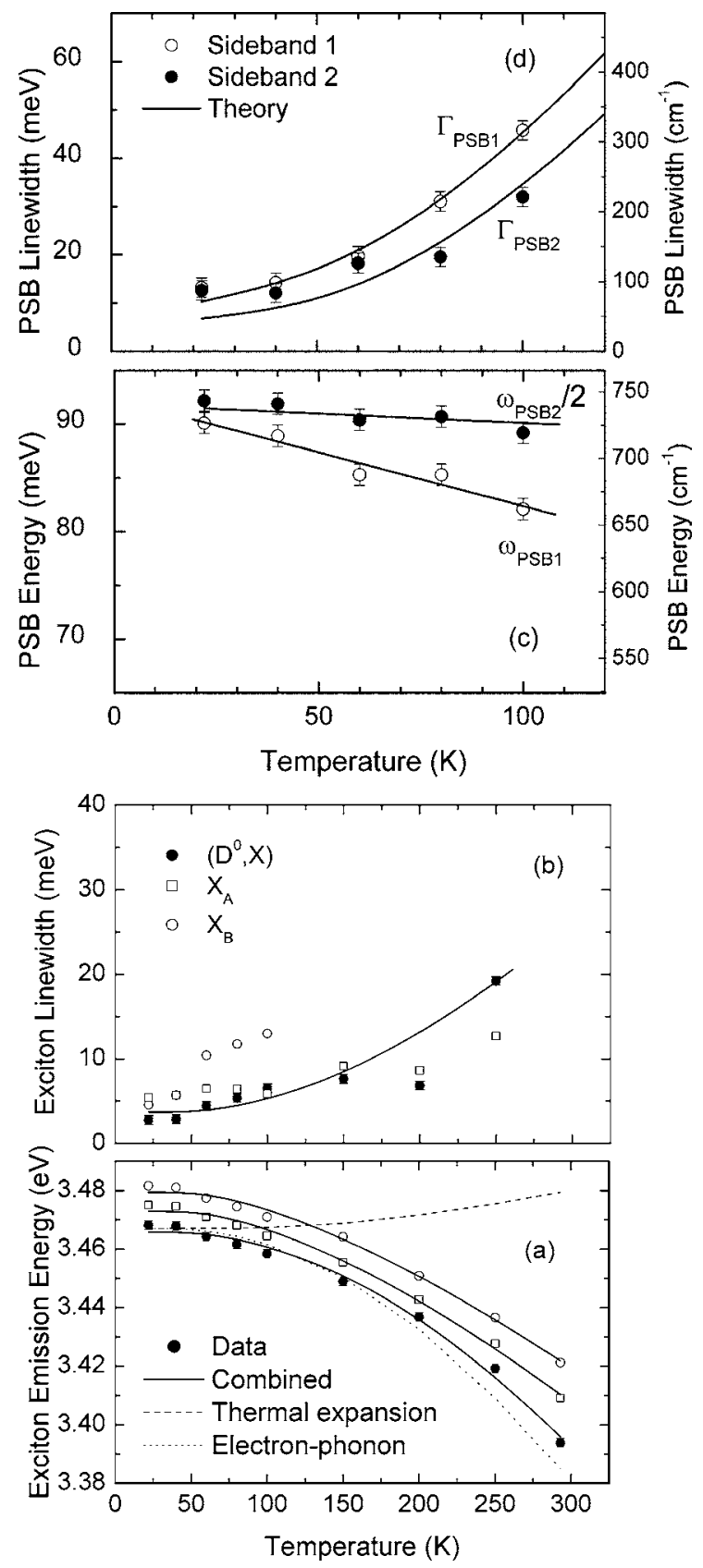

FIG. 4. Temperature dependences of exciton energies (a) and linewidths (b). Temperature dependences of LO-phonon PSB energies (c) and linewidths (d). The second PSB energy $\omega_{\mathrm{PSB} 2}$ is scaled by a factor of 2 so that it can be directly compared with $\omega_{\mathrm{PSB} 1}$. Curves are theoretical fits to the data.

$$
\begin{aligned}
E(T)= & E(0)-\frac{\delta}{k_{B}}\left[\varepsilon_{a c} W_{a c} n\left(\varepsilon_{a c}, T\right)+\varepsilon_{\mathrm{opt}} W_{\mathrm{opt}} n\left(\varepsilon_{\mathrm{opt}}, T\right)\right] \\
& -\int_{0}^{T}\left[\xi_{c} \alpha_{c}\left(T^{\prime}\right)+2 \xi_{a} \alpha_{a}\left(T^{\prime}\right)\right] d T^{\prime}
\end{aligned}
$$

where the last term accounts for thermal expansion. ${ }^{29}$ Quantities $\xi_{a}$ and $\xi_{c}$ are deformation potentials in the $a$ and $c$ crystal directions, respectively. ${ }^{30}$

The temperature dependence of the band gap and the fit to data using Eq. (4) are shown in Fig. 4(a). The four independent fitting parameters in Eq. (4) are also related to the exciton broadening discussed below. Table I summarizes the fit results. We break out the various contributions to the shift from the electron-phonon interaction $\Delta E_{e-p}(T)$. The majority of the temperature dependence stems from the electronphonon contribution. Our results show the two-phonon Pässler approach accurately describes the temperature dependence of the GaN band gap. The phonon energies in the model are $\sim 23 \mathrm{meV}=186 \mathrm{~cm}^{-1}$ and $\sim 69 \mathrm{meV}=557 \mathrm{~cm}^{-1}$, respectively, corresponding to weighted averages of the acoustic and optic density of states, respectively. ${ }^{26}$

The temperature dependence of the exciton linewidths, Fig. 4(b), is attributed to phonon-induced band broadening ${ }^{31}$

$$
\Gamma(T)=\Gamma(0)+W_{a c} \Gamma_{a c} n\left(\varepsilon_{a c}, T\right)+W_{\mathrm{opt}} \Gamma_{\mathrm{opt}} n\left(\varepsilon_{\mathrm{opt}}, T\right),
$$

where $\Gamma(0)$ is the inhomogeneous linewidth due to intrinsic lifetime, electron-electron interactions, defects, and strain. The second term in Eq. (5) arises from acoustic phonons and the third term is from optic phonons. In the intermediate temperature range, $\varepsilon_{a c}<k_{B} T<\varepsilon_{\text {opt }}$ we recover the less general approximation $n\left(\varepsilon_{a c}, T\right) \propto T .^{31} W_{a c}$ and $W_{\text {opt }}$ are fixed at the values obtained for the energy gap dependence, Eq. (4), and the phonon energies $\varepsilon_{a c}$ and $\varepsilon_{\mathrm{opt}}$ are likewise the values obtained from that analysis. $\Gamma_{a c}$ and $\Gamma_{\mathrm{opt}}$ are the electronphonon coupling constants. ${ }^{29}$ Treating $\Gamma(0), \Gamma_{a c}$, and $\Gamma_{\text {opt }}$ as fitting parameters, we obtain $\Gamma(0) \sim 3.7$ and $5.8 \mathrm{meV}$ for the $\left(D^{0}, X\right)$ and $X_{A}$ excitons, respectively. The intensity of the $X_{B}$ band decreases quickly with higher temperature. Below $T$ $=200 \mathrm{~K}$ its temperature dependence follows the same trend as the other exciton bands. We thus rely on $\left(D^{0}, X\right)$ and $X_{A}$ in obtaining fitting parameters. We obtain values for $\Gamma_{a c}$ $\sim 50 \mathrm{meV}$ and $\Gamma_{\text {opt }} \sim 110 \mathrm{meV}$. Direct comparison may be made with other studies, although composite quantities $W_{a c} \Gamma_{a c}$ and $W_{\text {opt }} \Gamma_{\text {opt }}$ are generally published as a single fitting parameter. The results are in good agreement with theoretical $^{32}$ values for the optic phonons, $60.2 \mathrm{meV}$ to compare with our $W_{\mathrm{opt}} \Gamma_{\mathrm{opt}}=55 \mathrm{meV}$. It is substantially lower than the previous experimental value $W_{\mathrm{opt}} \Gamma_{\mathrm{opt}}=100 \mathrm{meV},{ }^{11}$ which we attribute to the different methods used in describing the acoustic contribution to the linewidth and to the low dislocation density of our current materials.

\section{PHONON SIDEBANDS}

We now turn our attention to the temperature dependence of the $A_{1}$ (LO) PSBs in Fig. 4. Summarized in Figs. 4(c) and 4(d) are the PSB energies and linewidths, extending the range previously studied to below $50 \mathrm{~K} .{ }^{33}$ We graph energy $\omega_{\mathrm{PSB} 2} / 2$ for direct comparison with $\omega_{\mathrm{PSB} 1} . \Gamma_{\mathrm{PSB} 1}$ and $\Gamma_{\text {PSB2 }}$ are not rescaled. PSB properties have been studied theoretically ${ }^{24}$ and experimentally, ${ }^{33,34}$ with interesting results. The shift in $\omega_{\mathrm{PSB} 1}$ is considerably larger than for $\omega_{\mathrm{PSB} 2} / 2$. Further, $\Gamma_{\mathrm{PSB} 2}$ is found to be significantly narrower than $\Gamma_{\mathrm{PSB} 1}$. Prior work focused on the importance of the exciton-phonon density of states in the broadening and shift. The transition probability is $P_{m}(\omega) \propto \chi_{m}^{(5 / 2)-m} \exp \left(-\chi_{m} / k_{B} T\right)$, where $\chi_{m}=\hbar \omega+m \hbar \omega_{\mathrm{LO}}-E_{\mathrm{exc}}, m(=1,2)$ is the PSB index, and $E_{\mathrm{exc}}$ is the exciton energy. ${ }^{24}$ Physically, the prefactor originates from the density of states and the Boltzmann factor arises from the Fermi tail describing the thermal popula- 
tion of the exciton. This theory may be used to describe the PSB shift and linewidth as a function of temperature, ${ }^{33}$ but it does not account for the phonon broadening expected with increasing temperature. Including phonon broadening, we expect the energy to depend on temperature according to

$$
\omega_{\mathrm{PSB} m}(T)=m \omega_{0}-\left(\frac{5}{2}-m\right) k_{B} T-m \Delta_{2}(T),
$$

where $\omega_{0}$ and $\Delta_{2}(T)$ are for the $A_{1}$ (LO) phonon, Eqs. (1) and (2), taken directly from the Raman analysis. The linear term in $T$ arises from the shift in $P_{m}(\omega)$. Since $\omega_{\mathrm{PSB}}$ is relative to the exciton, the band gap shift of Eq. (4) is absent. Agreement between the data and Eq. (6) is excellent for both sidebands. The PSB shifts are dominated by terms linear in $T$ while the phonon decay plays a small role over this temperature range. The broadening exhibits significant curvature in Fig. 4(d), suggesting that the phonon-related processes are important in the dependence. The PSB widths also have a term linear in temperature. Using the Segall-Mahan theory and including the exciton and phonon broadening factors, the sideband linewidths vary according to

$$
\Gamma_{\mathrm{PSB} m}(T)=2 \sqrt{\left(\frac{5}{2}-m\right)} k_{B} T+\Gamma(T)+\Gamma_{P}(T),
$$

where $\Gamma(T)$ is from Eq. (5) and $\Gamma_{P}(T)$ is from Eq. (3). The latter term is negligible since the $\mathrm{A}_{1}(\mathrm{LO})$ band is very narrow compared to the other terms in Eq. (7). Fit results are included in Fig. 4(d). The $\Gamma(0)$ values of 6.0 and $4.5 \mathrm{meV}$ for $\mathrm{PSB}_{1}$ and $\mathrm{PSB}_{2}$ are close to the exciton linewidths at low temperature. The phonon energies for $\Gamma(T)$ are the same as what we obtained for the excitons in Table I. The exciton broadening parameters from the $X_{A}$ line are used. However, the temperature dependent term in Eq. (5) is ten times larger for the PSBs than for the excitons. This may be associated with the rapid rise seen in the $X_{B}$ linewidth below $100 \mathrm{~K}$, Fig. 4(b). Physically, this broadening may stem from the elevated electron-phonon interactions, attributed to quantum interference between intermediate states of excitons via the LO phonons. In GaN, the participating states are the separate exciton levels and these effects give rise to the asymmetric Fano line shape of the phonon sidebands. ${ }^{24,33,34}$

\section{SUMMARY}

In summary, we have investigated the influence of phonons on the temperature dependence of optical transitions in GaN. By comprehensively studying the phonon properties and the band gap-related emission we examine the relationship between vibrational and electronic properties. Both phonons and excitons are narrow, indicative of high-quality GaN. The observed band gap dependence is fully described based on the Fan and Debye-Waller factors using the approach of Pässler. ${ }^{26}$ Exciton broadening is also found to depend solely on the phonon band structure which is consistent with results of the energy gap dependence. The temperature dependence of the $A_{1}$ (LO) PSBs are described using the Segall-Mahan theory, ${ }^{24}$ with the phonons taken into account. We find the $A_{1}$ (LO) phonon properties play a minor role in the PSB energies, but play a strong role in the line broaden- ing. Our results are highly relevant to understanding the relationship between electronic bands and phonons in related semiconductors, particularly $\mathrm{AlN}$ and $\mathrm{InN}$ which have the wurtzite crystal structure but exhibit widely varying energy band structures.

\section{ACKNOWLEDGMENTS}

The authors thank S. Zollner for helpful discussions on the Debye-Waller factor. The authors acknowledge support by the National Science Foundation (ECS-0323640 and ECS-0304224) and the J. F. Maddox Foundation.

${ }^{1}$ P. G. Klemens, Phys. Rev. 148, 845 (1966).

${ }^{2}$ B. K. Ridley, J. Phys. Condens. Matter 8, L511 (1996).

${ }^{3}$ I. Ahmad, V. Kasisomayajula, M. Holtz, J. M. Berg, S. R. Kurtz, C. P. Tigges, A. A. Allerman, and A. G. Baca, Appl. Phys. Lett. 86, 173503 (2005).

${ }^{4}$ H. Morkoc, J. Mater. Sci.: Mater. Electron. 12, 677 (2001).

${ }^{5}$ N. Grandjean, B. Damilano, and J. Massies, J. Phys. Condens. Matter 13, 6945 (2001)

${ }^{6}$ M. Albrecht, I. P. Nikitina, A. E. Nikolaev, Y. V. Melnik, V. A. Dmitriev, and H. P. Strunk, Phys. Status Solidi A 176, 453 (1999).

${ }^{7}$ A. S. Usikov et al., in UV Solid-State Light Emitters and Detectors, edited by M. Shur and A. Zukauskas (Kluwer, Amsterdam, 2004).

${ }^{8}$ N. Faleev, I. Ahmad, M. Holtz, H. Temkin, and Y. Melnik, J. Appl. Phys. 98, 123508 (2005).

${ }^{9}$ M. Holtz, M. Seon, T. Prokofyeva, H. Temkin, R. Singh, F. P. Dabkowski, and T. D. Moustakas, Appl. Phys. Lett. 75, 1757 (1999).

${ }^{10}$ V. Yu. Davydov, Yu. E. Kitaev, I. N. Goncharuk, A. N. Smirnov, J. Graul, O. Semchinova, D. Uffmann, M. B. Smirnov, A. P. Mirgorodsky, and R. A. Evarestov, Phys. Rev. B 58, 12899 (1998).

${ }^{11}$ A. S. Zubrilov, S. A. Nikishin, G. D. Kipshidze, V. V. Kuryatkov, H. Temkin, T. I. Prokofyeva, and M. Holtz, J. Appl. Phys. 91, 1209 (2002).

${ }^{12}$ S. A. Nikishinet al., Appl. Phys. Lett. 75, 2073 (1999).

${ }^{13}$ A. Link, K. Bitzer, W. Limmer, R. Sauer, C. Kirchner, V. Schwegler, M. Kamp, D. G. Ebling, and K. W. Benz, J. Appl. Phys. 86, 6256 (1999).

${ }^{14}$ M. Giehler, M. Ramsteiner, P. Waltereit, O. Brandt, K. H. Ploog, and H. Obloh, J. Appl. Phys. 89, 3634 (2001).

${ }^{15}$ O. Martínez, M. Avella, J. Jimenez, B. Gerard, R. Cusco, and L. Artus, J. Appl. Phys. 96, 3639 (2004).

${ }^{16}$ H. Tang and I. P. Herman, Phys. Rev. B 43, 2299 (1991).

${ }^{17}$ R. R. Reeber and K. Wang, J. Mater. Res. 15, 40 (2000).

${ }^{18}$ J. Serrano, M. Cardona, T. M. Ritter, B. A. Weinstein, A. Rubio, and C. T. Lin, Phys. Rev. B 66, 245202 (2002).

${ }^{19}$ K. T. Tsen, D. K. Ferry, A. Botchkarev, B. Sverdlov, A. Salvador, and H. Morkoc, Appl. Phys. Lett. 72, 2132 (1998).

${ }^{20}$ T. Rufet al., Phys. Rev. Lett. 86, 906 (2001).

${ }^{21}$ X. B. Chen, J. Huso, J. L. Morrison, L. Bergman, and A. P. Purdy, J. Appl. Phys. 98, 026106 (2005).

${ }^{22}$ W. S. Li, Z. X. Shen, Z. C. Feng, and S. J. Chua, J. Appl. Phys. 87, 3332 (2000).

${ }^{23}$ Z. X. Liu, K. P. Korona, K. Syassen, J. Kuhl, K. Pakula, J. M. Baranowski, I. Grzegory, and S. Porowski, Solid State Commun. 108, 433 (1998).

${ }^{24}$ B. Segall and G. D. Mahan, Phys. Rev. 171, 935 (1968).

${ }^{25}$ R. B. Capaz, C. D. Spataru, P. Tangney, M. L. Cohen, and S. G. Louie, Phys. Rev. Lett. 94, 036801 (2005).

${ }^{26}$ R. Pässler, J. Appl. Phys. 89, 6235 (2001).

${ }^{27}$ H. Y. Fan, Phys. Rev. 82, 900 (1951).

${ }^{28}$ S. Gopalan, P. Lautenschlager, and M. Cardona, Phys. Rev. B 35, 5577 (1987).

${ }^{29}$ M. Munoz, F. H. Pollak, M. B. Zakia, N. B. Patel, and J. L. Herrera-Perez, Phys. Rev. B 62, 16600 (2000).

${ }^{30}$ H. Y. Peng, M. D. McCluskey, Y. M. Gupta, M. Kneissl, and N. M. Johnson, Phys. Rev. B 71, 115207 (2005).

${ }^{31}$ S. Rudin, T. L. Reinecke, and B. Segall, Phys. Rev. B 42, 11218 (1990).1098-0121

${ }^{32}$ M. Germainet al., J. Appl. Phys. 91, 9827 (2002).

${ }^{33}$ S. J. Xu, G. Q. Li, S. J. Xiong, S. Y. Tong, C. M. Che, W. Liu, and M. F. Li, J. Chem. Phys. 122, 244712 (2005).

${ }^{34}$ S. J. Xu, W. Liu, and M. F. Li, Appl. Phys. Lett. 77, 3376 (2000). 